\title{
High Speed/Low Dose Analytical Electron Microscopy with Machine Learning and Multi-Objective Dynamic Sampling
}

\author{
Karl A. Hujsak ${ }^{1}$, Eric Roth ${ }^{2}$, William Kellogg ${ }^{1}$, Lawrence F. Drummy ${ }^{3}$, and Vinayak P. Dravid ${ }^{1,2}$ \\ 1. Department of Materials Science \& Engineering, Northwestern University, Evanston, IL 60208 \\ 2. Electron Probe Instrumentation Center (EPIC) Facility, NUANCE Center, Northwestern University, \\ Evanston, IL 60208 \\ 3. Materials and Manufacturing Directorate, Air Force Research Laboratory, WPAFB, OH 45433
}

Newer generations of electron microscopes have dramatically increased spatial resolution and analytical sensitivity by focusing ever higher electron currents into smaller and smaller volumes. Atomic resolution imaging and atom-by-atom elemental mapping with Electron Energy Loss Spectroscopy (EELS) and Energy Dispersive X-ray Spectrometry (EDS) are being frequently reported for a wide variety of materials [1]. Commercially available MEMS-based sample holders, which can encapsulate the sample in a small volume of controllable fluid or gas, have also become widely available for most microscopes with accessible EDS or EELS signals. Radiation damage with higher available beam currents and the longer acquisition times for improved pixel resolution and field of view further exasperate an already complex problem of dose-induced damage/changes to ultrastructure of materials.

Combining EELS or EDS spectrometers with a mobile probe in a Scanning Transmission Electron Microscope (STEM) results in a spatial map of elemental signatures, referred to as a spectrum image. In contrast to energy filtered TEM, these spectrum images provide 'fine structure' which can be used to resolve bonding configuration and/or other information about local electronic structure. Unfortunately, as EDS and EELS are relatively dose-inefficient processes, they require long dwell times and consequently large electron doses for sufficient signal-to-noise ratios. Producing spectrum images with sufficient signal over large fields of view or with high pixel resolutions can often require hours, or even days. For many materials, especially soft or hybrid soft/hard materials, the accumulated dose can result in sample damage and loss of signal. This can be compounded in the case of in-situ studies where the presence of a dynamic medium can generate diffusing and damaging radical species. In addition, materials in liquids and gases are rarely stable and positionally static for long enough to record a highresolution spectrum image. These challenges necessitate innovative ways to conduct analytical studies that can reduce acquisition time yet retain requisite signal fidelity.

Since most of the pixels in an image do not contribute meaningful contrast, it has been proposed that randomly-under-sampling images and reconstructing them offline could suppress dose induced damage [2]. However, with a randomly selected mask, we have an equal chance of choosing less-informative and more informative measurements, and thus state of the art inpainting algorithms still require a minimum of $15-20 \%$ of the pixels to perform an effective reconstruction. Instead, we propose to actively identify the pixels which contribute meaningful information on-the-fly in STEM spectrum images, enabling an order of magnitude speed-up. Briefly, an initial 1\% of the grid points are acquired and used to construct a crude approximation of the true spectrum image for all elements present. Using features in the estimated image, such as gradients and local contrast variations, we use regression to estimate which potential measurements contain the most information [3]. This process is performed iteratively until the dose-threshold for the material is met or the estimated images of the sample converge. 
Our Multi-Objective Autonomous Dynamic Sampling method (MOADS) can effectively map multiple analytical signals for an arbitrary number of elements with over an order of magnitude less time compared to conventional raster scanning, and without complicated or computationally intensive inpainting methods. We demonstrate a facile interface to an arbitrary STEM using a Digital Micrograph $\left(\mathrm{DM}^{\circledR}\right.$, Gatan Inc.) plug in and its use in the mapping of multiple overlapping interfaces in EELS, as well as EDS spectrum images on dose sensitive biological materials. The use of a 'weakly informative' training database composed entirely of straight lines is employed to demonstrate its generalizability. This presentation will overview new additions to MOADS at ultra-low sampling rates, including methods for managing fluence and trade-offs between exploration and exploitation.

References:

[1] S.R. Spurgeon, Y. Du, S.A. Chambers. Microscopy and Microanalysis 23, no. 3 (2017): 513-517.

[2] K. Hujsak, B.D. Myers, E. Roth, Y. Li, and V. P. Dravid. $M \& M$ (2016): 1-11.

[3] G.M. Godaliyadda, D.H. Ye, M.D. Uchic, M.A. Groeber, G.T. Buzzard, and C.A.. Bouman.

Electronic Imaging 2016, no. 19 (2016): 1-8.

[4] This material is based on research sponsored by the Air Force Research laboratory under agreement No. FA8650-15-2-5518 and Air Force Office of Scientific Research under Award No. FA9550-

12-1-0280. This work made use of the EPIC facility of Northwestern University's NUANCE Center, which has received support from the Soft and Hybrid Nanotechnology Experimental (SHyNE) Resource (NSF ECCS-1542205); the MRSEC program (NSF DMR-1720139) at the Materials Research Center; the International Institute for Nanotechnology (IIN); the Keck Foundation; and the State of Illinois.

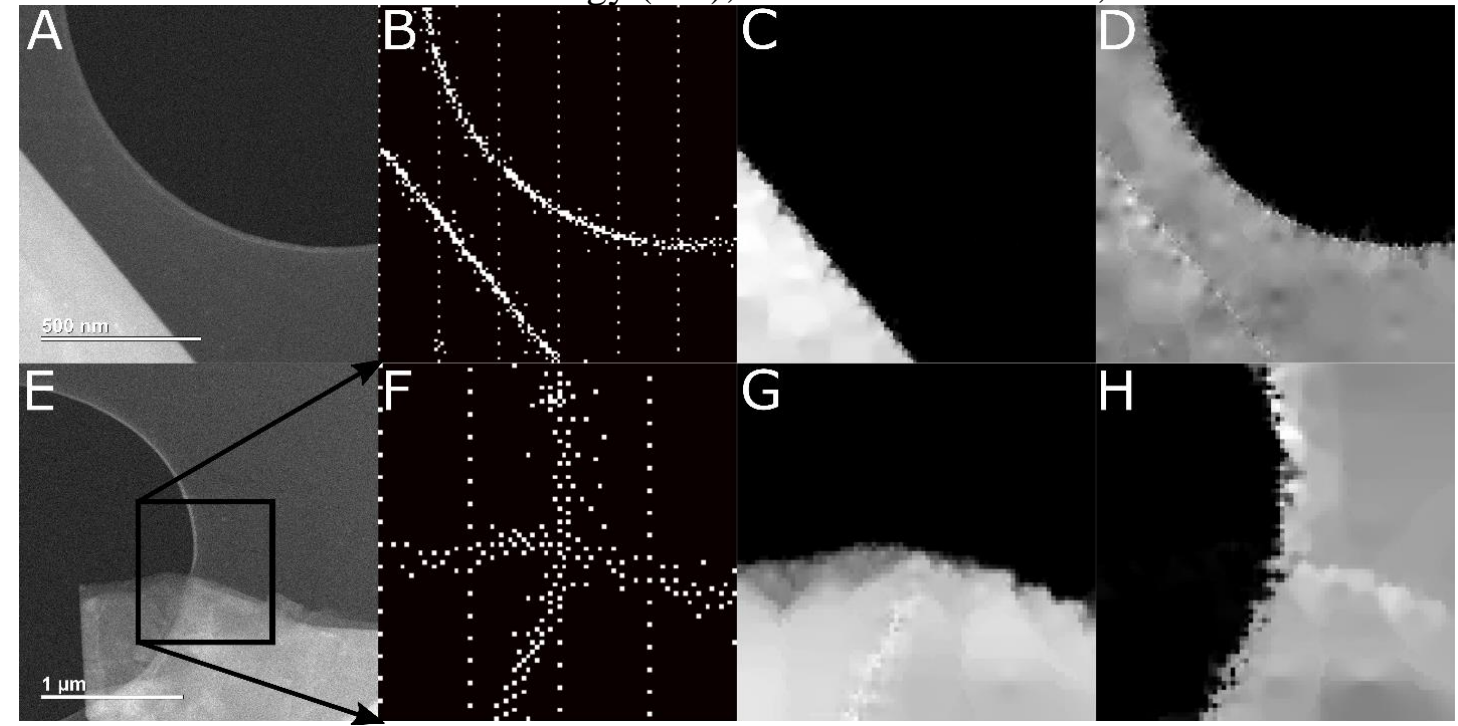

Figure 1. Experimental MOADS acquisition of boron nitride flakes on a lacey carbon film using Gatan Enfina EELS spectrometer. A. HAADF image of a boron nitride flake supported by carbon film. B. Mask of points collected by the microscope, white pixels representing sampled and black skipped. 4\% of the total pixels of the 120x120 spectrum image were collected in 9.6 minutes compared to 4 hours for a full spectrum image. C. Reconstructed boron map and D. reconstructed carbon map from the measured spectra in B. E. An additional area of boron nitride flake in which the boron signal overlaps with the carbon and the vacuum. F. Sampled spectra from the inset in E, 5\% of an $80 \times 80$ grid of spectra were collected in a total time of 7.5 minutes vs. 2.3 hours for a full spectrum image. G. Reconstructed boron and H. carbon maps. 